\title{
Alimentation lipidique et remplacement des huiles de poisson par des huiles végétales en pisciculture
}

Geneviève Corraze

Sadasivam Kaushik

Inra

UMR 1067 Nutrition, aquaculture

et génomique

Pôle d'hydrobiologie

64310 Saint-Pée-sur-Nivelle

France

$<$ kaushik@st-pee.inra.fr>

$<$ corraze@st-pee.inra.fr>

\begin{abstract}
Résumé
L'évolution des aliments piscicoles, au cours des dernières décennies, a été caractérisée par une augmentation de la teneur en lipides alimentaires afin d'améliorer les performances de croissance et de réduire les rejets azotés. Cette évolution, combinée à l'essor important de l'aquaculture, a conduit à une utilisation croissante d'huiles de poissons, dont la disponibilité est limitée. Le recours à des huiles végétales est donc une solution permettant un développement durable de l'aquaculture. L'utilisation d'aliments riches en lipides conduit aussi à une augmentation de l'engraissement des poissons au niveau du site préférentiel de stockage des lipides (foie, muscle, tissu adipeux périviscéral) qui varie selon les espèces. L'incorporation d'huiles végétales dans les aliments piscicoles, en remplacement de l'huile de poisson, n'altère pas les performances de croissance dans la mesure où les besoins en acides gras essentiels (AGE) sont couverts. En revanche, la composition en acides gras (AG) des poissons est fortement modifiée. Elle reflète en grande partie celle des huiles utilisées, avec en particulier une réduction des teneurs en acides gras poly-insaturés à longue chaîne AGPI-LC) de la série n-3 (EPA et DHA) qui, justement, confèrent aux poissons leur valeur diététique. Une alimentation de finition, avec des aliments à base d'huile de poisson, permet de restaurer les teneurs en ces AG dans la chair et donc de préserver sa valeur nutritionnelle. Cette possibilité de modulation de la composition en AG des poissons par l'alimentation est une stratégie qui devrait se généraliser pour permettre un développement durable de l'aquaculture préservant les ressources marines.
\end{abstract}

Mots clés : alimentation des poissons ; huile végétale ; lipide ; qualité.

Thèmes : alimentation, consommation, nutrition ; pêche et aquaculture ; productions animales; recherche scientifique et agronomique.

\section{Abstract \\ Lipid nutrition and fish oil replacement by vegetable oils in pisciculture}

Over the last few decades, changes in aquafeed have been characterised by an increase in dietary lipid levels to reduce nitrogen wastes and improve growth performance. This evolution combined with the strong increase in pisciculture production has led to a rise in demand for fish oils, while their availability remains limited. The use of vegetable oils would thus appear as an alternative which could contribute to reducing the part of marine resources in aquafeed. The use of high fat diets, however, leads to an increase in fat deposition in fish with changes occurring in the main storage site (liver, muscle, perivisceral adipose tissue) depending on the species of fish. Replacement of fish oil by vegetable oils does not affect growth performance if essential fatty acid requirements are met, whereas the fatty acid composition of the fish is strongly modified. Fatty acid composition of fish flesh generally reflects the fatty acid composition of the oils used with a decrease in $\mathrm{n}-3$ long chain polyunsaturated fatty acids (EPA and DHA) which confer to the flesh its nutritional value. A finishing period with diets based on fish oil restores the levels of these FA in the flesh, maintaining its nutritional value for human consumption. This possibility of tailoring fatty acid composition through dietary supply is important for sustainable pisciculture development and preservation of natural marine resources.

Key words: fish feeding; lipids; plant oils; quality.

Subjects: animal productions; fishing and aquaculture; food, consumption, nutrition; scientific and agricultural research. 
A u cours des 25 dernières années, l'évolution de la composition des aliments piscicoles a été caractérisée par une diminution de la teneur en protéines associée à une augmentation de l'apport lipidique, dans le but de réduire les rejets azotés tout en améliorant les performances de croissance. Cet effet d'épargne des protéines a été clairement démontré chez de nombreuses espèces ; cependant, l'utilisation de ces aliments " haute énergie " induit une augmentation des dépôts lipidiques corporels qui peut avoir des répercussions sur la qualité des produits (Corraze, 1999).

Par ailleurs, l'essor de l'aquaculture dans le monde (+10\% par an) se traduit par l'utilisation croissante d'aliments composés, dont les apports protéiques et lipidiques sont fournis essentiellement par les farines et les huiles de poissons. La stagnation des captures de pêche limite la disponibilité de ces matières premières. Ainsi, en 2003, l'aquaculture mondiale a utilisé, respectivement, 53 et $87 \%$ des productions de farines et huiles de poissons (Tacon, 2005). Afin de préserver les ressources naturelles tout en permettant un développement durable de l'aquaculture, il est donc impératif de diminuer l'emploi de ces ingrédients d'origine marine. Pour cela, il est nécessaire de diversifier les matières premières incorporées dans les aliments aquacoles, en particulier en utilisant des protéines et huiles végétales, disponibles en plus grande quantité et avec plus de régularité. Cependant, la nature des lipides incorporés dans les aliments conditionne la composition en acides gras (AG) de la chair. Or, la préservation de la teneur en acides gras polyinsaturés (AGPI) n-3 dans la chair des poissons reste l'objectif primordial, surtout pour l'alimentation de l'homme.

Cet article fait le point sur les recherches réalisées dans le domaine de la nutrition lipidique des poissons en relation avec la qualité des produits d'aquaculture.

\section{Influence du taux de lipides alimentaires}

Depuis les premiers travaux de Lee et Putnam (1973), de nombreuses études ont montré que l'augmentation de la teneur en lipides des aliments, et par voie de conséquence la diminution du rapport protéines/énergie digestible des aliments, permet une amélioration des performan- ces de croissance et une réduction des rejets azotés. L'évolution des aliments aquacoles a donc été caractérisée par une diminution sensible du taux de protéines alimentaires (de 50 à moins de $40 \%)$ avec une augmentation conséquente des lipides (de 12-15\% à plus de $30 \%$ pour les salmonidés, par exemple). L'effet d'épargne des protéines par les lipides a été montré chez de nombreuses espèces telles que la truite arc-en-ciel (Cho et Kaushik, 1990 ; Corraze, 1999), le saumon atlantique (Hillestad et Johnsen, 1994 ; Einen et Roem, 1997), le bar (Boujard et al., 2004), la daurade (Santinha et al., 1999), la morue (Morais et al., 2001) ou le flétan (Berge et Storebakken, 1991).

Cependant, certaines espèces marines comme le turbot, la plie ou le pagre tolèrent mal des taux de lipides alimentaires supérieurs à $15 \%$, et on observe, dans ce cas, une réduction des performances de croissance (Regost et al., 2001a ; Oku et Ogata, 2000). De même, chez des espèces omnivores ou tropicales d'eau douce, une augmentation trop importante de la teneur en lipides alimentaires peut avoir aussi un effet négatif sur la croissance, comme cela a été montré chez le tilapia avec des aliments contenant plus de 12$15 \%$ de lipides (Hanley, 1991; Chou et Shiau, 1996), chez la carpe commune et la carpe chinoise pour des aliments contenant, respectivement, plus de 15 et $10 \%$ de lipides (Shimeno et al., 1995 ; Du et al., 2005).

L'utilisation des aliments " haute énergie ", riches en lipides, tend à favoriser l'engraissement chez les poissons d'élevage. En effet, si une partie des lipides apportés par l'alimentation sert de substrat pour la fourniture d'énergie "à la place " des protéines, une autre partie est " retenue " sous forme de dépôts corporels. Ainsi, de nombreuses études ont montré que les régimes à forte teneur en lipides conduisent à des modifications de la composition corporelle, caractérisées par un accroissement des lipides corporels accompagné d'une diminution de la teneur en eau, sans variation de la teneur en protéines (Watanabe, 1982 ; Corraze, 1999). Les différents compartiments corporels ne réagissent pas de la même façon. En effet, chez les poissons, le stockage des lipides peut avoir lieu dans plusieurs tissus : foie, muscle, tissu adipeux périviscéral et parfois tissu adipeux sous-cutané (Corraze, 1999). L'importance de ces sites de stockage varie selon les espèces : tissu péri- viscéral et - dans une moindre mesure muscle chez les salmonidés, foie chez les espèces marines comme la morue ou le bar, tissu adipeux sous-cutané chez le turbot.

Chez la truite arc-en-ciel, les teneurs en lipides musculaires et surtout celle des lipides viscéraux augmentent avec l'élévation du taux de lipides alimentaires, en revanche, la teneur en lipides hépatiques ne varie pas (tableau 1). Les lipides stockés dans le tissu adipeux périviscéral sont en grande partie responsables de l'élévation des lipides corporels. Ainsi, lorsque le contenu en lipides des aliments passe de 15 à $30 \%$, la contribution des viscères et du muscle à l'élévation des lipides corporels est, respectivement, de 63 et $37 \%$ (Gélineau et al., 2001). Le même type de réponse a également été observé chez la truite fario (Regost et al., 2001b). Ces données confirment donc le rôle prépondérant de ces deux tissus comme sites de stockage des lipides chez la truite. De même, chez la carpe, la teneur en lipides des viscères augmente de 10,8 à $16,7 \%$, avec des régimes contenant respectivement 7 et $15 \%$ de lipides, mais la teneur en lipides musculaires varie peu (1,4 à 1,6\%) (Watanabe et al., 1987). Chez le saumon atlantique recevant des aliments à très forte teneur en matières grasses (jusqu'à $30 \%$ ), outre l'élévation des lipides viscéraux, on observe également une augmentation de la teneur en lipides musculaires qui passe de 8,5 à $12 \%$ pour des aliments contenant, respectivement, 21 et $32 \%$ de lipides (Hillestad et Johnsen, 1994).

En revanche, chez les poissons marins comme le bar, ce sont essentiellement les lipides hépatiques qui augmentent et, dans une moindre mesure, ceux des viscères, la teneur en lipides du muscle variant peu (figure 1). On observe une augmentation de $30 \%$ de la teneur en lipides du foie et de $13 \%$ dans les viscères pour des aliments contenant, respectivement, 15 et $30 \%$ de lipides (Boujard et al., 2004). De même, chez le turbot, lorsque le taux lipidique des aliments augmente de 10 à $20 \%$, on observe une élévation des lipides hépatiques (de 14 à $21 \%$ ), mais pas de modifications de la teneur en lipides du muscle, très faible chez cette espèce (1 à 1,5\%), ni de celle des viscères (Regost et al., 2001a). Il faut noter que chez cette espèce une partie des lipides est accumulée au niveau sous-cutané (4\%). 


\section{Tableau 1. Effets du taux de lipides alimentaires sur la composition corporelle et la teneur en lipides tissulaires chez la truite arc-en-ciel (d'après Gélineau et al., 2001).}

Table 1. Effect of dietary lipid level on body composition and tissue lipid contents in rainbow trout (Gélineau et al., 2001).

\begin{tabular}{lcccc}
\hline Lipides (\%) & $\mathbf{1 5}$ & $\mathbf{2 0}$ & $\mathbf{2 5}$ & $\mathbf{3 0}$ \\
\hline IHS & $1,2 \pm 0,2$ & $1,1 \pm 0,0$ & $1,2 \pm 0,1$ & $1,1 \pm 0,0$ \\
IVS & $9,1 \pm 0,0^{\mathrm{b}}$ & $10,1 \pm 0,5^{\mathrm{b}}$ & $12,0 \pm 0,0^{\mathrm{a}}$ & $12,9 \pm 0,9^{\mathrm{a}}$ \\
Composition corporelle & & & & \\
Eau (\%) & $65,9 \pm 0,1^{\mathrm{a}}$ & $65,6 \pm 0,2^{\mathrm{a}}$ & $63,3 \pm 0,1^{\mathrm{b}}$ & $62,0 \pm 1,0^{\mathrm{b}}$ \\
Lipides (\%) & $13,5 \pm 0,7^{\mathrm{b}}$ & $13,7 \pm 0,1^{\mathrm{b}}$ & $17,0 \pm 0,7^{\mathrm{a}}$ & $18,4 \pm 2,2^{\mathrm{a}}$ \\
Protéines (\%) & $17,3 \pm 0,7$ & $17,4 \pm 0,1$ & $16,7 \pm 0,7$ & $15,9 \pm 0,3$ \\
Lipides tissulaires (g/100 g tissu) & $5,2 \pm 0,4$ & $5,9 \pm 1,2$ & $7,4 \pm 0,2$ & $7,5 \pm 1,0$ \\
Foie & $51,1 \pm 0,5^{\mathrm{b}}$ & $52,5 \pm 2,4^{\mathrm{b}}$ & $59,1 \pm 1,6^{\mathrm{a}}$ & $59,9 \pm 0,8^{\mathrm{a}}$ \\
Viscères & $8,8 \pm 0,1^{\mathrm{b}}$ & $9,9 \pm 0,3^{\mathrm{b}}$ & $11,2 \pm 0,5^{\mathrm{a}, \mathrm{b}}$ & $13,3 \pm 1,4^{\mathrm{a}}$ \\
Muscle & & & & \\
\hline
\end{tabular}

Les valeurs représentent la moyenne $\pm \mathrm{ET}(n=6)$. Les valeurs qui ne sont pas affectées d'une même lettre sont significativement différentes $(p<0,05)$. IHS : indice hépatosomatique: (poids foie/poids poisson)/100; IVS: indice viscérosomatique: (poids viscères + tissu adipeux périviscéral/poids poisson)/100.

D’une façon générale, chez toutes les espèces, c'est le site préférentiel de stockage des lipides qui subit l'influence la plus marquée, à savoir le foie pour les espèces marines et le tissu adipeux périviscéral pour les espèces d'eau douce.
L'augmentation des lipides tissulaires ne se répercute pas de la même façon sur toutes les classes lipidiques: la teneur en phospholipides reste presque constante, ce sont les lipides neutres (surtout les triglycérides) qui sont responsables de

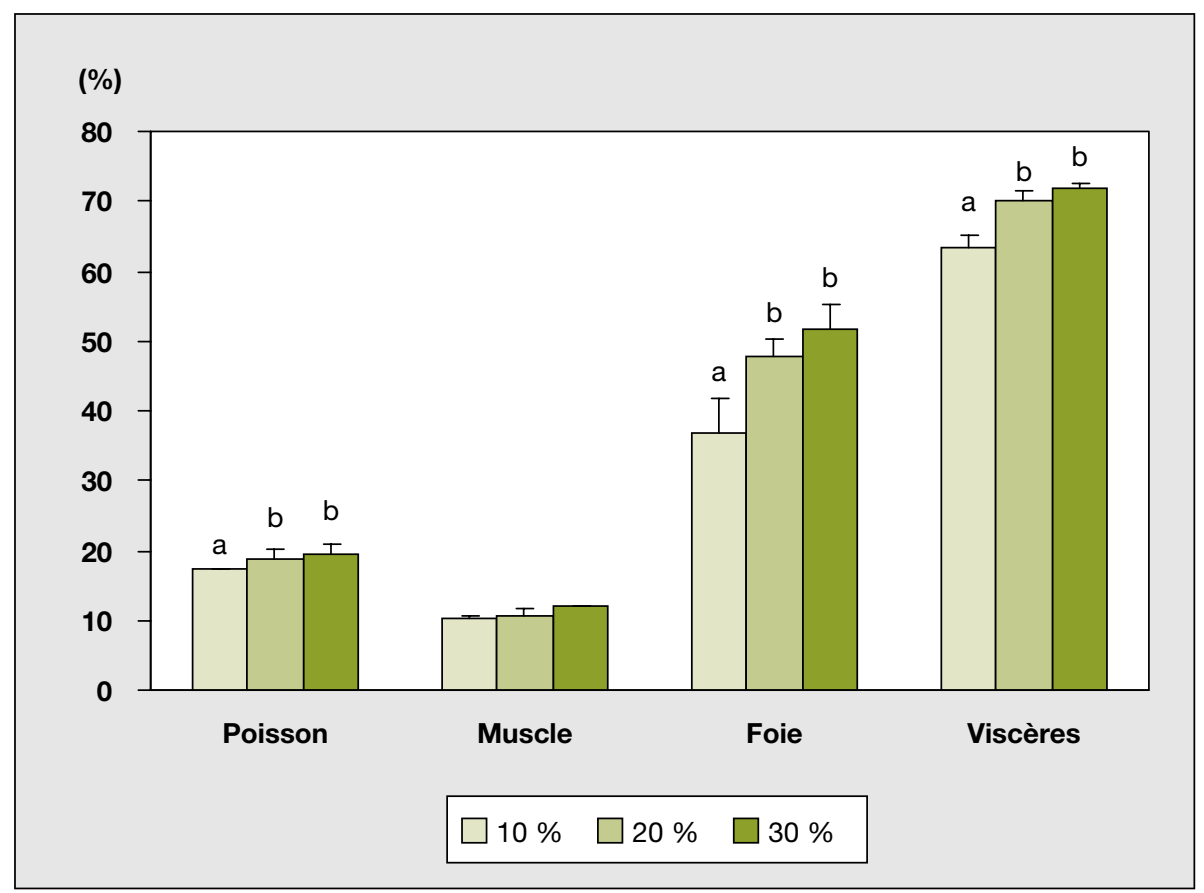

Figure 1. Teneurs en lipides corporels chez le bar en fonction de la teneur en lipides alimentaires (Boujard et al., 2004).

Figure 1. Body lipid content in sea bass in relation to dietary lipid levels (Boujard et al., 2004).

la quasi-totalité de l'accroissement observé (Corraze, 1999).

Ces variations de la teneur en lipides peuvent avoir des conséquences non seulement sur la valeur marchande et la qualité des produits frais, mais aussi sur l'aptitude à la transformation. Ainsi, chez les salmonidés, un développement excessif des dépôts lipidiques périviscéraux constitue une perte de rendement préjudiciable pour les transformateurs, les viscères pouvant représenter de 7 à $22 \%$ du poids vif. De même, pour les produits destinés au fumage, même si une certaine teneur en lipides de la chair est nécessaire, de trop forts taux induisent des pertes au cours du traitement qui peuvent aller jusqu'à 20 à $25 \%$ du poids du filet. En revanche, l'augmentation de la teneur en lipides de la chair semble avoir peu d'impact sur les qualités sensorielles des produits (Rasmussen, 2001) ; cependant, ces résultats demeurent encore controversés.

Chez toutes les espèces, la teneur en lipides des aliments influence donc les performances de croissance et conditionne l'état d'engraissement des poissons. Les aliments utilisés actuellement en aquaculture contiennent des teneurs en lipides comprises entre 19 et $30 \%$ pour les salmonidés en grossissement, 16 à $22 \%$ pour les poissons marins comme le bar ou la daurade et 10 à $16 \%$ pour les poissons d'étang.

\section{Remplacement des huiles de poisson par des huilles végétales}

La principale source lipidique utilisée en alimentation des poissons est l'huile de poisson qui apporte les acides gras polyinsaturés à longue chaîne (AGPI-LC) de la série n-3, en particulier l'EPA (acide eicosapentaénoïque: 20:5 n-3) et le DHA (acide docosahexaénoïque: 22:6 n-3). Compte tenu de l'utilisation d'aliments à forte teneur en énergie et de l'essor de l'aquaculture mondiale, la disponibilité en huile de poisson est devenue très limitée, et il convient de trouver des substituts. C'est pourquoi les recherches se sont orientées sur les possibilités de remplacement de l'huile de poisson par des huiles végétales, disponibles en plus grande quantité et avec plus de régularité. Les principales huiles végétales produites au niveau mondial sont les huiles de soja et de palme qui représentent plus de $55 \%$ 
du marché, mais également les huiles de colza et de tournesol (respectivement 14 et $9 \%$ des volumes produits en 2006). Cependant, la composition en AG des huiles végétales est très différente de celle des huiles de poisson. Elles sont dépourvues d'AGPI-LC n-3 et contiennent des proportions élevées d'AG de la série n-6 et n-9 (en particulier les acides linoléique : 18:2 n-6 et oléique : 18:1 n-9), mais aussi d'AG saturés (16:0 en particulier pour l'huile de palme). Certaines d'entre elles contiennent des proportions assez importantes d'acide alphalinolénique (18:3 n-3) ; c'est le cas pour l'huile de colza (8-10\%), mais surtout pour l'huile de lin (plus de $50 \%$ ).

Compte tenu de ces différences de composition, l'incorporation d'huiles végétales peut donc avoir des répercussions sur la croissance et la qualité nutritionnelle des produits

Les lipides alimentaires doivent permettre de couvrir les besoins en acides gras essentiels (AGE) qui sont différents en fonction des espèces. Les poissons d'eau douce sont capables, à partir des AG en C18 apportés par l'alimentation, de synthétiser par le biais de réactions de désaturation et d'élongation des AGPI-LC (20:5 et 22:6 n-3), ce qui n'est pas le cas des poissons marins. Cette quasiincapacité de bioconversion des AG est due à l'absence ou à une activité très faible de certaines enzymes impliquées dans ces réactions, en particulier les désaturases. Ces différences interspécifiques entraînent de grandes différences sur le besoin en AGE. Pour les poissons d'eau douce, ce besoin est de l'ordre de $1 \%$ de 18:3 n-3 ou de $0,5 \%$ de 20:5 et 22:6 n-3, les AGPI longs étant plus efficaces pour couvrir le besoin. Chez les poissons marins, l'acide alphalinolénique est totalement inefficace pour la couverture du besoin en AGE, il faut impérativement que l'alimentation apporte 0,5 à $1 \%$ de 20:5 et 22:6 n-3 (Corraze, 1999).

De nombreuses études ont montré que, dans la mesure où les besoins en AG essentiels sont couverts par les lipides déjà contenus dans la farine de poisson, l'utilisation d'huiles végétales est possible sans effet délétère sur la croissance. Ainsi, chez les salmonidés, le remplacement partiel (jusqu'à $80 \%$ ), voire total, de l'huile de poisson par des huiles végétales (colza, lin ou tournesol) ou par un mélange d'huiles végétales (palme + colza + lin) ne modifie pas les performances de croissance (Bell et al., 2003, 2004; Caballero et al., 2002 ; Torstensen et al., 2004, 2005; Richard et al., 2006). De plus, chez ces mêmes espèces, il a été montré que la substitution est possible dès le stade alevin et pendant toute la durée du cycle d'élevage (Torstensen et al., 2005 ; Richard et al., 2006). Chez les poissons marins, dont les capacités de bioconversion du 18:3 n-3 en AGPI n-3 à longue chaîne sont plus limitées, une substitution jusqu'à $60 \%$ par des huiles végétales n'entraîne pas d'effet négatif sur la croissance chez le bar (Izquierdo et al., 2003 ; Mourente et al., 2005), la daurade (Izquierdo et al., 2005), le turbot (Regost et al., 2003), le pagre (Glencross et al., 2003) ou la sériole (Watanabe, 2002). À plus fort taux d'incorporation $(>80 \%$ ), un effet dépressif sur la croissance a, cependant, été mis en évidence chez le bar et la daurade (Izquierdo et al., 2003, 2005).

Cette substitution n'a pas de répercussions marquées sur la composition corporelle des poissons ni sur la teneur en lipides musculaires. En revanche, la composition en AG des tissus est fortement modifiée. Dans le cas du muscle, cela revêt une importance toute particulière, puisque c'est la richesse en AG n-3 de la chair qui confère aux poissons leur qualité nutritionnelle et leur valeur diététique. De nombreuses études ont montré que la composition en $A G$ des tissus reflète, dans une large mesure, celle des lipides alimentaires ingérés chez les salmonidés (Caballero et al., 2002; Bell et al., 2003, 2004; Corraze et al., 2004; Kaushik et Corraze, 2004 ; Torstensen et al., 2004, 2005) et d'autres espèces d'eau douce comme la carpe ou le poisson-chat (Steffens et al., 1995; Ng et al., 2003), mais aussi chez les poissons marins (Glencross et al., 2003; Regost et al., 2003 ; Izquierdo et al., 2003, 2005 ; Montero et al., 2005 ; Mourente et al., 2005). L'incorporation d'huiles végétales dans les aliments, en remplacement de l'huile de poisson, entraîne une augmentation des AG caractéristiques des huiles végétales (18:1, 18:2 n-6, 18:3 n-3) dans les lipides tissulaires et une réduction du 20:5 et 22:6 n-3, AG spécifiques des huiles de poisson (figures 2 et 3). Ainsi, chez le saumon atlantique, après 50 semaines d'alimentation avec des régimes contenant de l'huile de colza ou de l'huile de lin, la proportion de 18:2 n- 6 dans la chair est de 12 à $14 \%$ contre seulement $4 \%$ chez les saumons nourris avec un régime à base d'huile de poisson. La teneur en 18:3 n-3 est très élevée (37 \%) dans la chair des animaux ayant reçu l'huile de lin, alors qu'elle n'est que de 1,5\% avec le régime contenant l'huile de poisson. À l'inverse, les proportions d'EPA et de DHA dans la chair sont réduites, 1,5 et $5,5 \%$ respectivement, avec les huiles végétales, comparées à 4 et $13 \%$ avec l'huile de poisson (Bell et al., 2003) (figure 3). L'utilisation de mélanges d'huiles végétales au lieu de

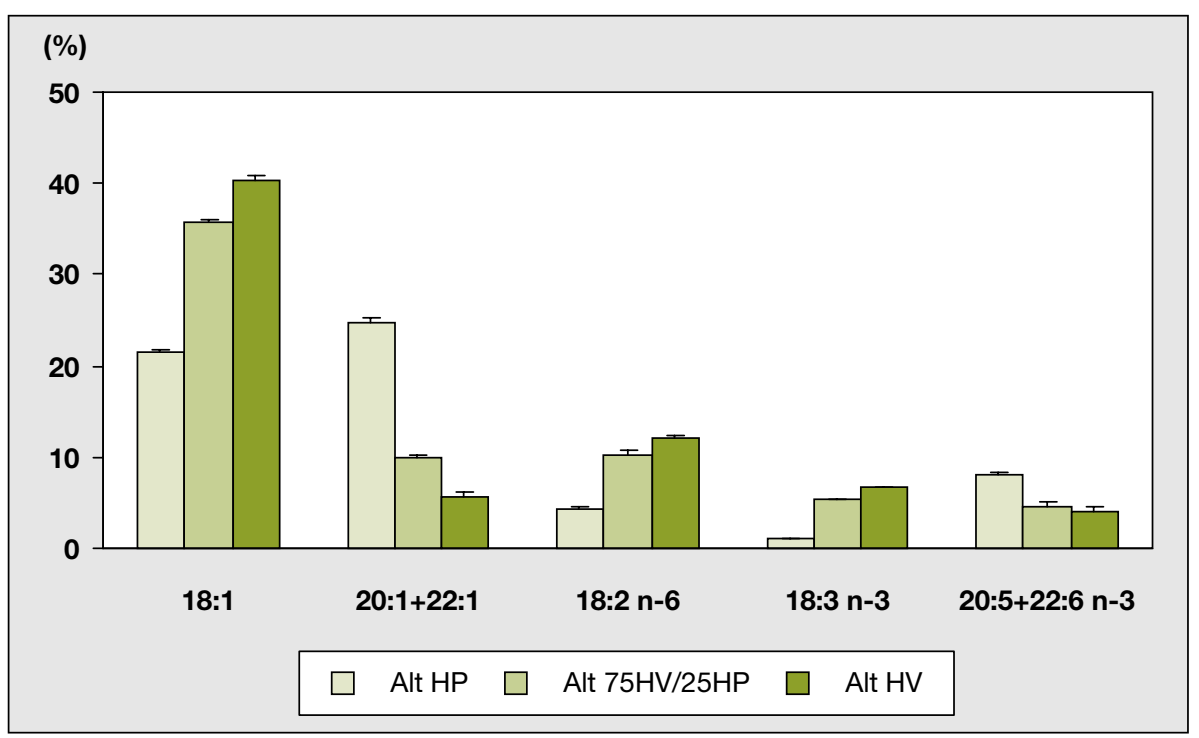

Figure 2. Composition en acides gras des lipides neutres du muscle chez la truite arc-en-ciel ayant reçu des régimes contenant $100 \%$ d'huile de poisson, $75 \%$ d'huile végétales $/ 25 \%$ d'huile de poisson ou $100 \%$ d'huiles végétales pendant 62 semaines (pourcentage acides gras totaux) (Corraze et al., 2004).

Figure 2. Fatty acid composition of muscle neutral lipids in rainbow trout fed diets containing $100 \%$ fish oil, $75 \%$ vegetable oils/ $25 \%$ fish oil or $100 \%$ vegetable oils for 62 weeks (\% total FA) (Corraze et al., 2004). HP : huile de poisson ; HV : mélanges d'huiles végétales. 


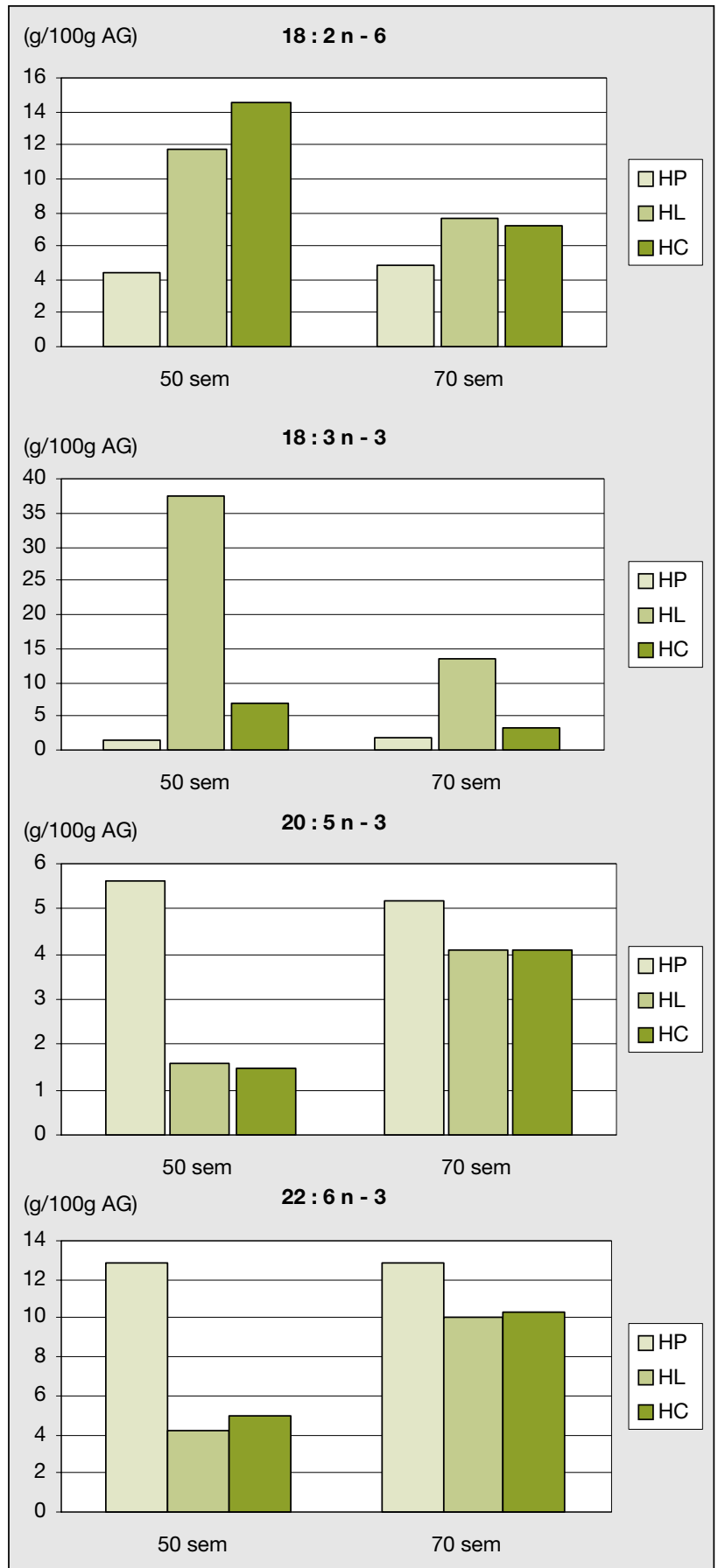

Figure 3. Composition en acides gras des lipides totaux de la chair de saumon après des régimes alimentaires contenant $100 \%$ d'huile de poisson, $100 \% d^{\prime}$ huile de lin ou $100 \%$ d'huile de colza pendant 50 semaines et après 20 semaines supplémentaires d'alimentation avec un régime $100 \%$ huile de poisson (Bell et al., 2003).

Figure 3. Fatty acid contents of total lipid from salmon flesh after feeding diets containing $100 \%$ fish oil, $100 \%$ linseed oil or $100 \%$ rapeseed oil for 50 weeks and after a further 20 weeks of feeding a $100 \%$ fish oil diet (Bell et al., 2003).

HP : huile de poisson; HL : huile de lin ; HC : huile de colza.

sources uniques d'huile permet de minimiser les variations de composition en $A G$ de la chair, en particulier pour le 18:1, 18:2 n-6 et 18:3 n-3. Par exemple, chez la truite arc-en-ciel après 62 semaines d'alimentation avec un mélange d'huiles végétales composé de $55 \%$ de colza, $30 \%$ de palme, $15 \%$ de lin, les propor- tions de 18:3 n-3 et de 18:2 n-6 dans les lipides musculaires augmentent de 1 à $6,6 \%$ et de 4,4 à $12 \%$, respectivement, par rapport à celles des animaux ayant reçu un régime à base d'huile de poisson (Corraze et al., 2004).

Les différences de composition en AG dans les tissus en fonction du type d'huiles ingérées sont cependant de moindre importance que celles observées dans les aliments. En particulier, les teneurs en AGPI n-3 sont supérieures dans les tissus, comparées à celles dans les aliments, ce qui indique une rétention sélective de certains AG (DHA et, dans une moindre mesure, EPA) et/ou une synthèse de ces AG à partir des précurseurs à chaîne plus courte (C18). Les modifications de composition en AG portent essentiellement sur la fraction lipides neutres, la composition des phospholipides, principaux composants des membranes cellulaires, étant en général plus stable. Ainsi, l'influence de la nature des lipides alimentaires sur les lipides tissulaires sera fonction de la teneur en lipides et du ratio lipides neutres/phospholipides. Ces données ont permis d'établir un modèle prédictif de dilution des AG corporels par les AG alimentaires (Robin et al., 2003). La corrélation avec la composition des aliments sera d'autant plus forte que la teneur en lipides neutres des tissus (essentiellement les triglycérides) sera élevée (Sargent et al., 2002 ; Robin et al., 2003).

Les modifications de composition en AG de la chair n'ont pas de répercussion sur ses qualités organoleptiques. Ainsi, même avec une substitution totale de l'huile de poisson par des huiles végétales, aucune modification notable des paramètres d'évaluation sensorielle (aspect, odeur, flaveur, texture) n'est observée, et ce, quelle que soit l'espèce (Regost et al., 2003 ; Corraze et al., 2004 ; Izquierdo et al., 2005 ; Torstensen et al., 2005 ; Montero et al., 2005).

Ces études montrent que la nature des lipides incorporés dans les aliments conditionne en grande partie le profil en AG de la chair. En effet, même chez les espèces ayant des capacités importantes de bioconversion des AG comme les salmonidés, les teneurs en AGPI-LC n-3 dans la chair sont fortement réduites lors de l'incorporation d'huiles végétales.

Pour préserver la richesse en AGPI-LC n-3 de la chair des poissons, et donc sa valeur diététique, il est possible de recourir à une alimentation de finition. Des travaux récents ont ainsi montré qu'il est possible de restaurer des teneurs élevées en EPA et 
DHA dans la chair des poissons ayant reçu au préalable des huiles végétales, en nourrissant les animaux quelques mois avant l'abattage avec un aliment à base d'huile de poisson. Chez le saumon atlantique ayant reçu pendant 50 semaines des aliments contenant $100 \%$ d'huile de lin ou de colza, une période d'alimentation de finition de 20 semaines permet d'obtenir des teneurs en EPA et DHA équivalentes à $80 \%$ de celles de saumons ayant reçu un aliment à base d'huile de poisson pendant tout le cycle d'élevage (Bell et al., 2003) (figure 2). Des résultats similaires ont été obtenus après alimentation avec un mélange d'huiles végétales pendant tout le cycle d'élevage, puis une période de finition de trois ou six mois chez la truite arc-en-ciel et le saumon, avec une restauration de l'EPA à environ $70 \%$ et du DHA à 80-90\% (Richard, 2006 ; Torstensen et al., 2005). De même, chez le bar ou la daurade ayant reçu des aliments contenant $60 \%$ d'huiles végétales, la teneur en DHA de la chair des poissons est restaurée à $100 \%$, et celle de l'EPA à 70-80 \%, après une période de finition de 22 ou 12 semaines, respectivement (Montero et al., 2005; Izquierdo et al., 2005). Chez la plupart des espèces, il est plus facile de restaurer les teneurs en DHA que celles en EPA ; cela est probablement lié au fait que l'EPA est préférentiellement oxydé, comparé au DHA et/ou que le DHA est préférentiellement incorporé dans les lipides tissulaires (Montero et al., 2005 ; Izquierdo et al., 2005).

Lors de cette période de finition, on observe en parallèle une diminution des teneurs en acides linoléique (18:2 n-6) et alphalinolénique (18:3 n-3), caractéristiques des huiles végétales, dans la chair des poissons, mais ces valeurs restent toujours plus élevées, surtout pour le 18:2 n6, que dans la chair d'animaux alimentés uniquement avec des huiles de poisson.

\section{Conclusion}

L'ensemble de ces études montre la grande plasticité de la composition en AG de la chair des poissons. Une période d'alimentation de finition, de l'ordre de 12 semaines en fin de cycle d'élevage, permet de maintenir la valeur nutritionnelle de la chair des poissons et donc son intérêt pour la nutrition humaine tout en limitant l'utilisation des huiles de poisson pour l'aquaculture. Cette straté- gie permet donc de préserver les ressources marines tout en assurant un développement durable de l'aquaculture.

\section{Références}

Bell JG, Tocher DR, Henderson RJ, Dick JR, Crampton VO. Altered fatty acid compositions in atlantic salmon (Salmo salar) fed diets containing linseed and rapeseed oils can be partially restored by a subsequent fish oil finishing diet. J Nutr $2003 ; 133$ : 2793-801.

Bell JG, Henderson RJ, Tocher DR, Sargent JR. Replacement of dietary fish oil with increasing levels of linseed oil: modification of flesh fatty acid compositions in atlantic salmon ( $\mathrm{Sal}$ mo salar) using a fish oil finishing diet. Lipids 2004 ; 39 : 223-32.

Berge GM, Storebakken T. Effect of dietary fat level on weight gain, digestibility and fille composition of atlantic halibut. Aquaculture $1991 ; 99: 331-8$.

Boujard T, Gelineau A, Coves D, et al. Regulation of feed intake, growth, nutrient and energy utilisation in European sea bass (Dicen trarchus labrax) fed high fat diets. Aquaculture $2004 ; 231: 529-45$

Caballero MJ, Obach A, Rosenlund G, Montero D, Gisvold M, Izquierdo MS. Impact of different dietary lipid sources on growth, lipid digestibility, tissue fatty acid composition and histology of rainbow trout, Oncorhynchus mykiss. Aquaculture $2002 ; 214$ : 253-71.

Cho $\mathrm{CY}$, Kaushik SJ. Nutritional energetics in fish: protein and energy utilization in rainbow trout. In : Bourne GH, ed. Aspects of food production, consumption and energy values. World Rev Anim Nutr 1990; 61 : 132-72.

Chou BS, Shiau SY. Optimal dietary lipid level for growth of juvenile hybrid tilapia, Oreochromis niloticus $X$ Oreochromis aureus. Aquaculture $1996 ; 143: 185-95$

Corraze G. Nutrition lipidique. In : Guillaume $J$ Métailler R, Bergot $P$, Kaushik SJ, eds. Nutrition des poissons et des crustacés. Paris: Inra éditions, 1999.

Corraze G, Radunz-Neto J, Richard N, Cardinal M, Kaushik S. Remplacement de l'huile de poisson par des huiles végétales chez la truite arc-en-ciel: incidence sur les qualités nutritionnelles et organoleptiques. Viandes et Produits Carnés 2004 ; (HS) : 115-6.

Du ZY, Liu YJ, Tian LX, Wang JT, Wang Y, Liang GY. Effect of dietary lipid level on growth, feed utilisation and body composition by juvenile grass carp (Ctenopharyngodon $i$ della). Aquacult Nutr $2005 ; 11: 139-46$.

Einen O, Roem AJ. Dietary protein/energy ratios for atlantic salmon in relation to fish size: growth, feed utilization and slaughter quality. Aquacult Nutr $1997 ; 3: 115-26$.

Gélineau A, Corraze G, Boujard T, Larroquet L, Kaushik S. Relation between dietary lipid level and voluntary feed intake, growth, nutrient gain, lipid deposition and hepatic lipogenesis in rainbow trout. Reprod Nutr Dev 2001; 41 : 487-503.

Glencross BD, Hawkins WE, Curnow JG. Evaluation of canola oils as alternative lipid resources in diets for juvenile red sea bream, Pagrus auratus. Aquacult Nut 2003 ; 9 : 409-18.

Hanley F. Effects of feeding supplementary diets containing varying levels of lipid on growth, food conversion and body composition of Nile tilapia, Oreochromis niloticus (L.). Aquaculture 1991 ; 93 : 323-34.
Hillestad M, Johnsen F. High energy/low protein diets for atlantic salmon: effects on growth, nutrient retention and slaughter quality. Aquaculture 1994 ; 124 : 109-16.

Izquierdo MS, Montero D, Robaina L, Caballero MJ, Rosenlund G, Gines R. Alterations in fillet fatty acid profile and flesh quality in gilthead sea bream (Sparus aurata) fed vegetable oils for a long term period. Recovery of fatty acid profiles by fish oil feeding. Aquaculture 2005 ; $250: 431-44$.

Izquierdo MS, Obach A, Arantzamendi L, Montero D, Robaina L, Rosenlund G. Dietary lipid sources for sea bream and sea bass: growth performance, tissue composition and flesh quality. Aquacult Nutr $2003 ; 9$ : 397-407.

Kaushik SJ, Corraze G. Substitution des huiles de poisson dans les aliments pour poisons. AquaFilia 2004 ; 2 : 5-9.

Lee DJ, Putnam GB. The response of rainbow trout of varying protein-energy ratios in a test diet. J Nutr 1973; 3 : 916-22.

Montero D, Robaina L, Caballero MJ, Ginés R, Izquierdo MS. Growth, feed utilization and flesh quality of European sea bass (Dicentrarchus labrax) fed diets containing vegetable oils: A time-course study on the effect of arefeeding period with a $100 \%$ fish oil diet. Aquaculture 2005 ; 248 : 121-34.

Mourente G, Good JE, Bell JG. Partial substitution of fish oil with rapeseed, linseed and olive oils in diets for European sea bass (Dicentrarchus labrax L.): effects on flesh fatty acid composition, plasma prostaglandins $\mathrm{E}_{2}$ and $\mathrm{F}_{2 \alpha}$, immune function and effectiveness of a fish oil finishing diet. Aquacult Nutr 2005; 11: 25-40.

Morais S, Bell JG, Robertson DA, Roy WJ, Morris PC. Protein/lipid ratios in extruded diets for atlantic cod (Gadusmorhua L.): effects on growth, feed utilisation, muscle composition and liver histology. Aquaculture $2001 ; 203: 101-19$

Ng W, Lim P, Boey P. Dietary lipid and palm oil source affects growth, fatty acid composition and muscle $\alpha$-tocopherol concentration of African catfish, Clarias gariepinus. Aquaculture $2003 ; 215: 229-43$.

Oku H, Ogata H. Body lipid deposition in juveniles of red sea bream Pagrus major, yellowtail Seriola quinqueradiata, and Japanese flounder Paralichthys olivaceus. Fish Sci 2000; 66: 25-31.

Rasmussen RS. Quality of farmed salmonids with emphasis on proximate composition, yield and sensory characteristics. Aquac Res $2001 ; 32$ : 767-86.

Regost C, Arzel J, Cardinal M, Robin J, Laroche M, Kaushik S. Dietary lipid level, hepatic lipogenesis and flesh quality in turbot (Psetta maxima). Aquaculture 2001a ; 193 : 291-309.

Regost C, Arzel J, Cardinal M, Laroche M, Kaushik SJ. Fat deposition and flesh quality in seawater reared triploid brown trout ( $\mathrm{Sal}$ mo trutta) as affected by dietary fat levels and starvation. Aquaculture 2001b ; 193 : 325-45.

Regost C, Arzel J, Robin J, Rosenlund G, Kaushik SJ. Total replacement of fish oil by soybean or linseed oil with a return to fish oil in turbot (Psetta maxima) -1. Growth performance, flesh fatty acid profile, and lipid metabolism. Aquaculture 2003 ; 217 : 465-82.

Richard N. Effet du taux et de la nature des lipides alimentaires sur les mécanismes intervenant dans la constitution des dépôts lipidiques (transport, captage, synthèse) chez la truite arc-en-ciel et le bar. Thèse doctorat, université Bordeaux-I, Bordeaux, 2006. 
Richard N, Kaushik S, Larroquet L, Panserat S Corraze G. Replacing dietary fish oil by vegetable oils has little effects on lipogenesis lipid transport and tissue lipid uptake in rain bow trout (Oncorhynchus mykiss). Br J Nutr 2006 ; 96 : 299-309.

Robin JH, Regost C, Arzel J, Kaushik SJ. Fatty acid profile of fish following a change in die tary fatty acid source: model of fatty acid composition with a dilution hypothesis. Aquaculture $2003 ; 225$ : 283-93.

Santinha PJM, Médale F, Corraze G, Gomes EFS. Effects of the dietary protein: lipid ratio on growth and nutrient utilization in gilthead seabream (Sparus aurata L.). Aquacul Nut 1999 ; 5 : 147-56.

Sargent JR, Tocher DR, Bell JG. The lipids. In : Halver JE, Hardy RW, eds. Fish nutrition. 3rd ed. San Diego (California) : Academic Press, 2002.
Shimeno S, Kheyyali D, Shikata T. Metabolic response to dietary lipid to protein ratios in common carp. Fish Sci $1995 ; 61$ : 977-80.

Steffens W, Wirth M, Rennert B. Effects of adding various oils to the diet on growth, feed conversion and chemical composition of carp (Cyprinus carpio). Arch Anim Nutr 1995 ; $47: 381-9$.

Tacon AGJ. Salmon aquaculture dialogue: status of information on salmon aquaculture feed and the environment. International Aquafeed $2005 ; 8: 22-37$.

Torstensen BE, Frøyland L, Lie Ø. Replacing dietary fish oil with increasing levels of rapeseed oil and olive oil - effects on atlantic salmon (Salmo salar L.) tissue and lipoprotein lipid composition and lipogenic enzyme activities. Aquacult Nutr 2004 ; 10 : 175-92.
Torstensen BE, Bell JG, Rosenlund G, et al. Tailoring of atlantic salmon (Salmo salar L.) flesh lipid composition and sensory quality by replacing fish oil with a vegetable oil blend. JAgric Food Chem 2005; 53: 10166-78.

Watanabe T. Lipid nutrition in fish. Comp Bio chem Physiol 1982 ; 73B : 3-15

Watanabe T, Takeuchi T, Satoh S, Ida T, Yaguchi M. Development of practical carp diets for reduction of total nitrogen loading on water environment. Nippon Suisan Gakkai Shi $1987 ; 53: 1413-23$

Watanabe T. Strategies for further development of aquatic feeds. Fish Sci 2002; 68: 242-52. 